\title{
A Simple Method to Measure Graphite Thickness with Monolayer Precision using Plasmon Energy Loss Imaging
}

\author{
M. Boese, S. Kumar, M. Lotya, A. O’Neill, H. Z. Zhang, J. N. Coleman, G. S. Duesberg \\ * CRANN, Trinity College Dublin, Ireland
}

At present graphene - a single plane of sp2-hybridised carbon atoms - appears to be among the most studied materials. Most production methods however yield multiple layers of graphene. The measurement of thicknesses and exact determination of the number of graphene layers is therefore important to optimize and control the synthesis conditions $[1,2,4]$.

TEM can be used in bright field imaging mode to provide the numbers of layers [2]. Using diffraction is not an effective method of producing results. Sample tilt influences the intensity of the reflections and interpretation is hindered. Other methods like STEM/EELS [3] and AFM [2] use two-dimensional mapping. Data acquisition and post-processing are time consuming.

The method described here is using an Electron Spectroscopic Imaging approach. Since the probability of inelastic scattering increases with sample thickness, energy loss electrons are useful for imaging sample thickness. In particular, plasmon excitations have high cross sections. In this study an energy window is used to select the plasmon excitation signal for imaging. The plasmon peak provides sufficient signal to image the sample, even in live mode during TEM grid examination. The number of graphene layers is determined from grey-value analysis in the energy filtered image.

Fig. 1a is acquired with a primary electron energy of $80 \mathrm{kV}$. The average energy loss is $25 \mathrm{eV}$ within a window of $15 \mathrm{eV}$. This energy is selected to avoid the influence of the different energies caused by surface and bulk plasmon contributions. The resulting histogram Fig. 1b represents the grey values of Fig.1a. The combination of the grey value in the vacuum and the incremental decrease caused by a single flake enables the identification of a single graphene layer.

The comparison of bright field imaging and plasmon imaging is shown in Fig. 2a and Fig. 2b. The main advantage of the energy filtered image is the signal-to-noise ratio. Due to this high thickness sensitivity further interpretation of the results is possible:

The different roughness of the surfaces is clearly visible and caused by the different synthesis methods of the graphite (Fig. 1 and Fig. 2). The increase in brightness of the single layer, compared to the incremental constant of a single layer within the multilayers can be interpreted by contamination or reaction of the surface and is subject to further investigations.

\section{References}

[1] M. Lotya et al., J. Am. Chem. Soc. 131 (2009) 3611.

[2] Y. Hernandez et al., Nat. Nanotechnol. 3 (2008) 563.

[3] M.H. Gass et al., Nat. Nonotechnol. 3 (2008) 676.

[4] Kumar et al. Chemical Communications 46 (2010) 1422. 
[5] This work was conducted under the framework of the INSPIRE program, funded by the Irish Government's Programme for Research in Third Level Institutions, Cycle 4, National Development Plan 2007-2013

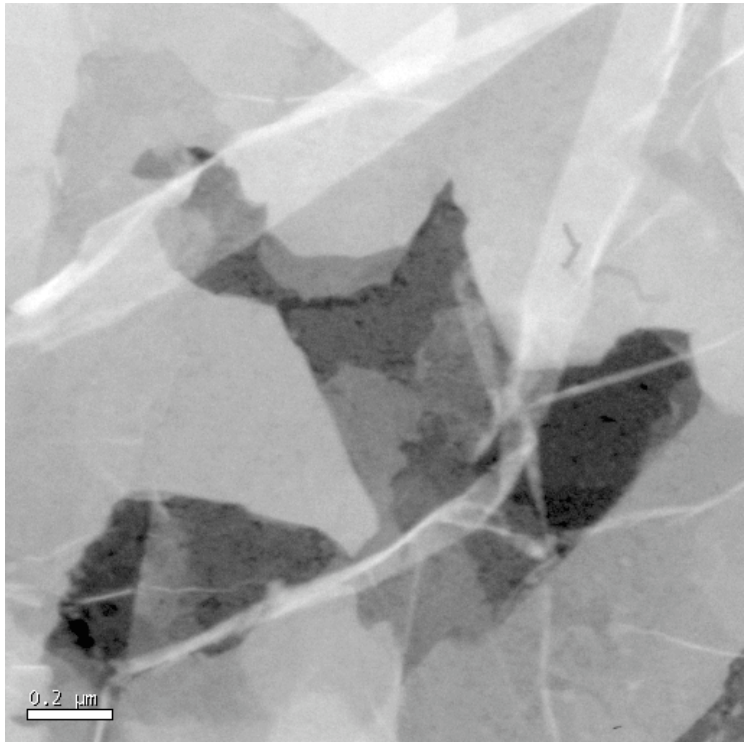

FIG. 1a. Plasmon energy loss filtered image of freestanding graphite film

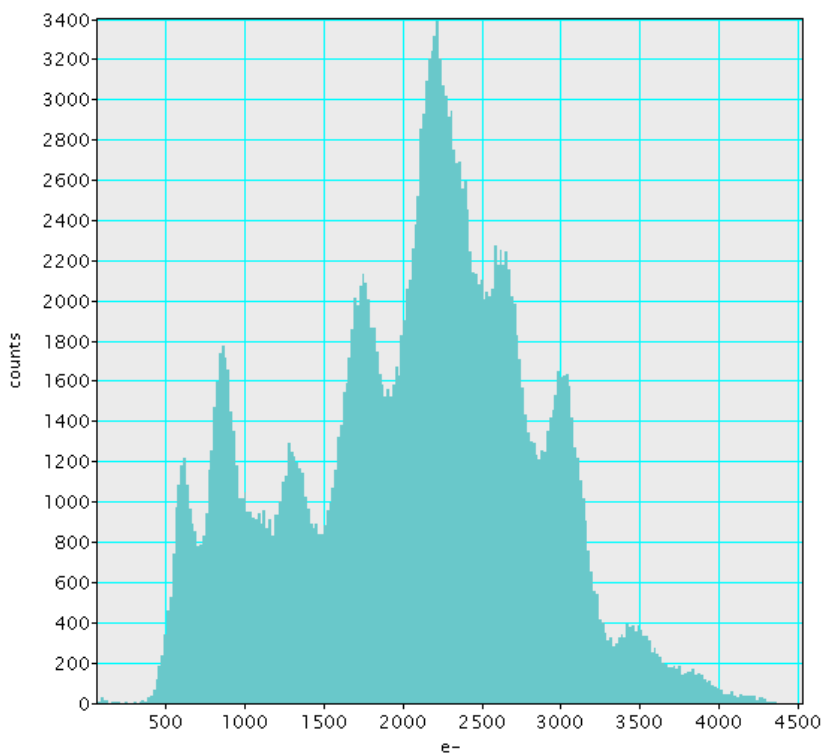

FIG. 1b. Histogram from 1a) showing most frequent grey value for 5 layers. First peak corresponds to a single layer of graphene
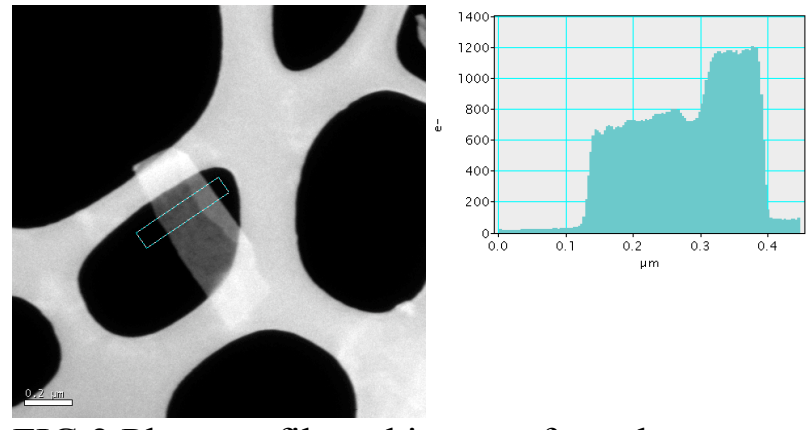

FIG.2 Plasmon filtered image of graphene flake and intensity profile across the flake
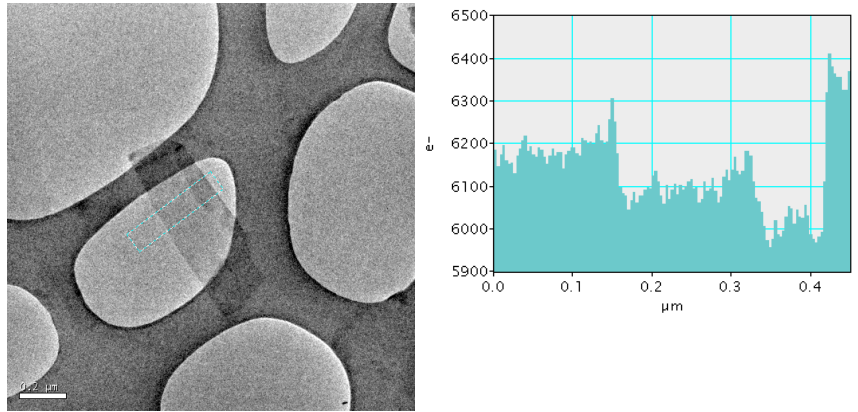

FIG.3 Bright field image of graphene flake and intensity profile across the flake 